\title{
Antibacterial Activity of Bees Gut Lactobacilli against Paenibacillus Larvae In Vitro
}

\author{
Miroslava Kačániová ${ }^{1,2^{\star}}$, Jaroslav Gasper ${ }^{1}$, Margarita Terentjeva ${ }^{3}$, Simona Kunová ${ }^{4}$, \\ Maciej Kluz', Czeslaw Puchalski ${ }^{2}$ \\ ${ }^{1}$ Slovak University of Agriculture, Faculty of Biotechnology and Food Sciences, Department of \\ Microbiology, Tr. A. Hlinku 2, 94976 Nitra, Slovakia \\ ${ }^{2}$ University of Rzeszow, Faculty of Biology and Agriculture, Department of Bioenergy and Food \\ Technology 35-601 Rzeszow, Zelwerowicza St. 4, Poland \\ ${ }^{3}$ Latvia University of Agriculture, Faculty of Veterinary Medicine, Institute of Food and Environmental \\ Hygiene K. Helmana iela 8, LV-3004, Jelgava, Latvia \\ ${ }^{4}$ Slovak University of Agriculture, Faculty of Biotechnology and Food Sciences, Department of Food \\ Hygiene and Safety, Tr. A. Hlinku 2, 94976 Nitra, Slovakia
}

\begin{abstract}
The aim of this study was to evaluate antimicrobial activity of bees gastrointestinal Lactobacillus spp. of against Paenibacillus larvae. Content of the intestinal tract was cultured for isolation of Lactobacillus spp. Gut homogenates were plated on de Man, Rogosa and Sharpe agar (MRS, Oxoid) plates and incubated for $48-72 \mathrm{~h}$ at $30^{\circ} \mathrm{C}$ anaerobically. Then, the identification of isolates with MALDI-TOF MS Biotyper was done. The bacterial strains Lactobacillus gasseri, L. amylovorus, L. kunkeei, L. fructivorans, Paenibacillus larvae were isolated from gut content of bees. The disc diffusion method was used for the determination of antimicrobial activities of the Lactobacillus supernatant against two strains of Paenibacillus larvae. The best antimicrobial activity of Lactobacillus against Paenibacillus larvae from gut was found in $L$. gasseri supernatant. Lesser degree of antimicrobial activity against $P$. larvae was found in $L$. kunkeei supernatant. The strongest antibacterial activity against $P$. larvae CCM 4438 was found in $L$. gasseri and $L$. amylovorus and the least antibacterial activity was found in $L$. fructivorans.
\end{abstract}

Keywords: in vitro, antibacterial effect, bees gut microflora, Paenibacillus larvae.

\section{Introduction}

Paenibacillus larvae is a Gram-positive, spore forming pathogen that causes the American foulbrood (AFB) in honey bee [1, 2]. P. larvae may form spore or exists as a vegetative cell depending on environmental conditions. For example, $P$. larvae forms resistant spores under nutrient deprivation with viability as long as 70 year [3]. P. larvae spores germinate only in the gut of honey bee larva host and pathogen needs

\footnotetext{
* Corresponding author: Miroslava Kačániová, miroslava.kacaniova@gmail.com
}

(cc) BY-NC-ND $\odot 2018$ Miroslava Kačániová et al., published by De Gruyte

This work was licensed under the Creative Commons Attribution-NonCommercialNoDerivs 3.0 License the honey bee larvae to complete the life cycle [2]. Newly germinated $P$. larvae proliferate with extreme bacteremia and death of the first or second instar larvae several days after infection [4]. P. larvae re-sporulate after consumption with formation of billions of spores, which are infectious spores are could be spread within the colonies by bees and beekeeping practices [5]. Gut bacteria stimulate the immunity of honey bee larvae, the stage, where organism is vulnerable to infection by different pathogens, and promote mounting of anti-pathogen immune responses [6]. Addition of probiotic bacteria into the larval food of honey bee leaded to the decreased the number of infected larvae with Paenibacillus larvae [7]. The aim of this study was to evaluate the effect of 
isolated gut bacteria from local honey bee Apis mellifera of Slovakia against two different strains of Paenibacillus larvae.

\begin{abstract}
Material and Methods
Collection of Apis mellifera and isolation of gut bacteria

Colonies of Apis mellifera from wooden hives in an apiary at the the East Slovakia in July 2017 were studied. Foragers $(n=10)$ and returning workers $(n=10)$ of $A$. mellifera from each colony were aseptically taken at hive entrances and put into a $1.5 \mathrm{ml}$ sterile tubes. For removal of gut, the digestive tracts were aseptically dissected, transferred into a tube with physiological solution and homogenized. Then the material was plated onto de Man, Rogosa and Sharpe agar (MRS, Oxoid) and incubated for $48-72 \mathrm{~h}$ at $30^{\circ} \mathrm{C}$. Bacterial colonies were identified according to size, colour and morphology [8].
\end{abstract}

\section{Sample preparation and MALDI-TOF MS measurement}

Prior to identification, the bacterial colonies were subcultured on Nutrition agar (Oxoid) at $37^{\circ} \mathrm{C}$ for 18-24 h. One colony of each bacterial isolate was selected for screening. Subsequently, an analysis of the bacteria identification was performed using the Maldi TOF MS Biotyper described by Kluga et al. [9].

\section{Bacterial strains for testing}

The bacterial strains Lactobacillus gasseri, L. amylovorus, $L$. kunkeei, $L$. fructivorans, Paenibacillus larvae were isolated from gut microflora of bees and Paenibacillus larvae CCM 4438 was collected from the Czech Collection of microorganisms (Brno, Czech Republic).

\section{Detection of antibacterial activity of lactobacilli}

Culture of Lactobacillus after 24h of incubation in MRS broth were centrifuged at $5500 \mathrm{~g}$ for $10 \mathrm{~min}$ at $4^{\circ} \mathrm{C}$ and $0.1 \mathrm{ml}$ of the supernatant was used for detection of antibacterial activity. The suspension of tested Paenibacillus larvae in saline $(0.1 \mathrm{ml}$ of $10^{5} \mathrm{cfu} / \mathrm{ml}$ ) was spread on Mueller Hinton Agar (MHA, Oxoid). Filter paper discs $(6 \mathrm{~mm}$ in diameter) were impregnated with $15 \mu \mathrm{l}$ of supernatant and placed on the inoculated agars. Agars were incubated at $4^{\circ} \mathrm{C}$ for $2 \mathrm{~h}$ and at $37^{\circ} \mathrm{C}$ for $24 \mathrm{~h}$. All the tests were performed in triplicate. Filter discs impregnated with a $10 \mu \mathrm{l}$ of distilled water were used as the negative, but antibiotic (amikacin $10 \mu \mathrm{g}$ and gentamicin $10 \mu \mathrm{g}$ ) as the positive control.

\section{Statistical analyses}

For Lactobacillus and Paenibacillus the mean and standard deviation of inhibition zones were calculated.

\section{Results and Discussions}

The composition of the gut bacterial communities of social insects has been shaped by coevolution. The social behaviour of honeybees provides favourable conditions for the exchange of the symbiont microbes [10] and these microorganisms are efficiently transmitted between bee colony members and their different generations. The gut microbial composition of insects, and especially honeybees, is unique, harbouring highly genetically diverse, niche-adapted bacterial species [11]. The model of symbiosis between honey bees and their bacterial microflora could inspire ideas of how to exploit this microflora for the protection of the host's health. The question of whether endogenous bacterial symbionts suppress honeybee pathogenic bacteria has been asked and analyzed for many times. However, no direct or clear evidence supporting this hypothesis has ever been found. The use of exogenous lactic acid bacteria $(\mathrm{LAB})$ as an alternative active flora has also been proposed [12,13]. Particular attention has been paid to testing exogenous $L A B$ strains that produce bacteriocins. The mortality of in vivo infected bee larvae was studied previously to investigate whether the gut bacteria ( $L$. kunkeei, P. mirabilis, E. kobei, M. morganii, F. fructosus, $B$. licheniformis, and $B$. subtilis) are capable of reducing the lethal effects of $P$. larvae infection [14].

In bees gut, four bacterial strains of Lactobacillus were isolated (Lactobacillus gasseri, $L$. amylovorus, L. kunkeei, L. fructivorans). From one bees colony Paenibacillus larvae was isolated. The scores of isolated bacteria showed in Table 1.

Table 1

MALDI-TOF scores of isolated microorganisms

\begin{tabular}{l|l}
\hline \multicolumn{1}{c|}{ Isolated bacteria } & Score \\
\hline Lactobacillus gasseri & 2.174 \\
Lactobacillus amylovorus & 2.206 \\
Lactobacillus kunkeei & 2.283 \\
Lactobacillus fructivorans & 2.076 \\
Paenibacillus larvae & 2.416 \\
\hline
\end{tabular}

The antibacterial activity of Lactobacillus against bees gut Peanibacillus larvae is shown in Table 2. 


\section{Antimicrobial activity of Lactobacillus supernatant against Paenibacillus larvae}

Table 2

\begin{tabular}{l|c|c}
\hline \multicolumn{1}{c|}{ Isolated bacteria } & Paenibacillus larvae from gut & Paenibacillus larvae CCM4438 \\
\hline Lactobacillus gasseri & $6.67 \pm 1.53$ & $5.67 \pm 1.53$ \\
Lactobacillus amylovorus & $6.00 \pm 1.00$ & $5.67 \pm 2.08$ \\
Lactobacillus kunkeei & $5.00 \pm 1.00$ & $5.33 \pm 0.58$ \\
Lactobacillus fructivorans & $5.33 \pm 1.53$ & $5.00 \pm 1.00$ \\
Amikacin & $10.97 \pm 0.49$ & $10.37 \pm 0.67$ \\
Gentamicin & $7.83 \pm 0.55$ & $7.53 \pm 0.06$ \\
\hline
\end{tabular}

The maximal antimicrobial activity was found for L. gasseri supernatant $(6.67 \pm 1.53 \mathrm{~mm})$. The minimal antimicrobial activity against $P$. larvae was found for $L$. kunkeei supernatant $(5.00 \pm 1.00$ $\mathrm{mm})$. The strongest antibacterial activity against $P$. larvae CCM 4438 was found for $L$. gasseri and L. amylovorus (5.67 \pm 1.53 resp. $5.67 \pm 2.08 \mathrm{~mm}$ ) and the weakest antibacterial activity for $L$. fructivorans supernatant $(5.00 \pm 1.00 \mathrm{~mm})$.

Our results were in accordance with Forsgren et al. [7], who demonstrated that the $L A B$, including $L$. kunkeei from honey bee gut, had strong inhibitory effect on in vitro growth of $P$. larvae.

\section{Conclusions}

Probiotic effects of four guts Lactobacillus isolated from the local honey bees of Slovakia were evaluated for their inhibitory activity against $P$. larvae. Lactobacillus gasseri, L. amylovorus, $L$. kunkeei and $L$. fructivorans can influence the growth of $P$. larvae isolated from bee. Bacteria belonging to Lactobacillus and some of their metabolites are getting significant importance in apiculture.

\section{Acknowledgements}

The study was funded by the European Community project No. 26220220180: Building Research Centre „AgroBioTech".

\section{References}

1. Alvarado, I., Margotta, J.W., Aoki, M.M., Flores, F., Agudelo, F., Michel, G., Elekonich, M.M., \& AbelSantos, E. (2017). Inhibitory effect of indole analogs against Paenibacillus larvae, the causal agent of American foulbrood disease. Journal of Insect Science, 17(5):104, 1-8, https://doi.org/10.1093/jisesa/iex080

2. Genersch, E. (2010). American Foulbrood in honeybees and its causative agent, Paenibacillus larvae. Journal of Invertebrate Pathology, 103, S10S19, https://doi.org/10.1016/i.jip.2009.06.015

3. Forsgren, E., Stevanovic J. \& Fries I. 2008. Variability in germination and in temperature and storage resistance among Paenibacillus larvae genotypes. Veterinary Microbiology, 129, 342-349, https://doi.org/10.1016/j.vetmic.2007.12.001
4. Lindström, A., Korpela, S. \& Fries, I. (2008). The distribution of Paenibacillus larvae spores in adult bees and honey and larval mortality, following the addition of American Foulbrood diseased brood or sporecontaminated honey in honey bee (Apis mellifera) colonies. Journal of Invertebrate Pathology, 99, 82-86, https://doi.org/10.1016/j.jip.2008.06.010

5. Yue, D., Nordhoff, M., Wieler, L. H. \& Genersch, E. (2008). Fluorescence in situ hybridization (FISH) analysis of the interactions between honeybee larvae and Paenibacillus larvae, the causative agent of American foulbrood of honeybees (Apis mellifera). Environmental Microbiology, 10, 1612-1620, https://doi.org/10.1111/j.1462-2920.2008.01579.x

6. Evans, J.D. \& Lopez D.L. (2004). Bacterial probiotics induce an immune response in the honey bee (Hymenoptera: Apidae). Journal of Economic Entomology, 97, 752-756.

7. Forsgren, E., Olofsson, T.C., V́asquez, A. \& Fries, I. (2010). Novel lactic acid bacteria inhibiting Paenibacillus larvae in honey bee larvae. Apidologie, 41, 99-108,

https://doi.org/10.1051/apido/2009065

8. Wu, M., Sugimura, Y., Iwata, K. Iwata, K., Takaya, N., Takamatsu, D. Kobayashi, M., Taylor, D., Kimura, K., \& Yoshiyama, M. (2014). Inhibitory Effect of Gut Bacteria from the Japanese Honey Bee, Apis cerana japonica, Against Melissococcus plutonius, the Causal Agent of European Foulbrood Disease. Journal of Insect Science. 14, 129, https://doi.org/10.1673/031.014.129

9. Klūga, A., Terentjeva, M., Kántor, A., Kluz, M., Puchalski, C., \& Kačániová, M. (2017). Antibacterial Activity of Melissa officinalis L., Mentha piperita L., Origanum vulgare $\mathrm{L}$. and Malva mauritiana against Bacterial Microflora Isolated from Fish. Advanced Research in Life Sciences, 1, 7580, https://doi.org/10.1515/arls-2017-0013

10. Engel, P. \& Moran, N.A. (2013). The gut microbiota of insects diversity in structure and function. FEMS Microbiology Reviews, 37, 699-735, https://doi.org/10.1111/1574-6976.12025

11. Engel, P. \& Moran, N.A. (2013). Functional and evolutionary insights into the simple yet specific gut microbiota of the honey bee from metagenomic analysis. Gut Microbes, 4, 60-65, https://doi.org/10.4161/gmic.22517

12. Yoshiyama, M., Wu, M., Sugimura, Y., Takaya, N., Kimoto-Nira, H. \& Suzuki, C. (2013). Inhibition of 
Paenibacillus larvae by lactic acid bacteria isolated from fermented materials, Journal of Invertebrate Pathology, 112, 62-67, https://doi.org/10.1016/i.jip.2012.09.002 13. Jaouani, I. Abbassi, M.S. Alessandria, V. Bouraoui, J. Ben Salem, R. Kilani, H. Mansouri, R. Messadi L. \& Cocolin L. (2014). High inhibition of Paenibacillus larvae and Listeria monocytogenes by Enterococcus isolated from different sources in Tunisia and identification of their bacteriocin genes. Letters of
Applied
Microbiology,
59 ,
$17-25$, https://doi.org/10.1111/lam.12239

14. Al-Ghamdi, A., Khalid, A.K., Ansari, M.J. Almasaudi, S.B., \& Al-Kahtani, S. (2017). Effect of gut bacterial isolates from Apis mellifera jemenitica on Paenibacillus larvae infected bee larvae. Saudi Journal of Biological Sciences, in press, https://doi.org/10.1016/j.sjbs.2017.07.005 